\title{
RANKS OF COMMUTATORS OF TRUNCATED TOEPLITZ OPERATORS ON FINITE DIMENSIONAL SPACES
}

\author{
Yong Chen, Kei Ji Izuchi And Young Joo LeE
}

Abstract. We study the rank of commutator $\left[A_{\eta}, A_{\eta}^{*}\right]$ of truncated Toeplitz operators $A_{\eta}$ and $A_{\eta}^{*}$ with several type of inner symbols $\eta$ on the model space $\mathscr{H}_{\theta}$ with finite Blaschke product $\theta$. Mathematics subject classification (2010): Primary 47B35; Secondary 32A37. Keywords and phrases: Truncated Toeplitz operator, model space, rank.

\section{REFERENCES}

[1] A. Baranov, R. Bessonnov And V. Kapustin, Symbols of truncated Toeplitz operators, J. Funct. Anal. 261 (2011), 3437-3456.

[2] A. Baranov, I. Chalendar, E. Fricain, J. Mashreghi and D. Timotin, Bounded symbols and reproducing kernel thesis for truncated Toeplitz operators, J. Funct. Anal. 259 (2010), 2673-2701.

[3] I. Chalendar and D. Timotin, Commutation relations for truncated Toeplitz operators, Oper. Matrices 8 (2014), 877-888.

[4] Y. Chen, H. Koo And Y. J. LeE, Ranks of complex skew symmetric operators and applications to Toeplitz operators, J. Math. Anal. Appl. 425 (2015), 734-747.

[5] J. Cima, S. GARCIA, W. Ross AND W. Wogen, Truncated Toeplitz operators: spatial isomorphism, unitary equivalence, and similarity, Indiana U. Math. J. 59 (2010) 595-620.

[6] J. Cima, W. Ross AND W. Wogen, Truncated Toeplitz operators on finite dimensional spaces, Oper. Matrices 2 (2008), 357-369.

[7] R. BRUCE CRofoot, Multipliers between invariant subspaces of the backward shift, Pacic J. Math. 166 (1994) 225-246.

[8] S. Garcia, J. Mashreghi And W. Ross, Introduction to Model Space and their Operators, Cambridge Studies in Advanced Mathematics, Volume 148, Cambridge University Press, 2016.

[9] S. GARCIA AND W. Ross, Recent progress on truncated Toeplitz operators, Fields Institute Communications 65 (2013), 275-319.

[10] E. Fricain, A. Hartmann And W. Ross, Multipliers between model spaces, Studia Math. 240 (2018), 177-191.

[11] D. SARASON, Algebraic properties of truncated Toeplitz operators, Oper. Matrices 1 (2007) 491-526.

[12] N. SEDLOCK, Algebras of truncated Toeplitz operators, Oper. Matrices 5 (2011), 309-326.

[13] E. Strouse, D. Timotin And M. ZARRABI, Unitary equivalence to truncated Toeplitz operators, Indiana Univ. Math. J. 61 (2012), 525-538. 